\title{
Efecto del nivel de suplementación con concentrado sobre la respuesta productiva en vacas lecheras a pastoreo primaveral con alta disponibilidad de pradera ${ }^{\#}$
}

\author{
Effect of the level of concentrate supplementation on the productive response of dairy cows on \\ spring grazing receiving a high herbage allowance \\ RG Pulido $^{a}$, A Escobar ${ }^{a}$, S Follert ${ }^{\mathrm{a}}$, M Leiva ${ }^{\mathrm{a}}$, P Orellana ${ }^{\mathrm{b}}$, F Wittwer ${ }^{\mathrm{c}}$, O Balocchid \\ ${ }^{a}$ Instituto de Ciencia Animal, Facultad de Ciencias Veterinarias, Universidad Austral de Chile, Valdivia, Chile. \\ ${ }^{\mathrm{b}}$ Departamento de Ciencias Pecuarias, Facultad Medicina Veterinaria, Universidad de Concepción, Concepción, Chile. \\ ${ }^{\mathrm{c}}$ Instituto de Ciencias Clínicas Veterinarias, Facultad de Ciencias Veterinarias, Universidad Austral de Chile, Valdivia, Chile. \\ ${ }^{\mathrm{d}}$ Instituto de Producción Animal, Facultad de Ciencias Agrarias, Universidad Austral de Chile, Valdivia, Chile.
}

\section{SUMMARY}

The purpose of this study was to evaluate the effect of different levels of concentrate supplementation on the productive response of dairy cows fed high daily herbage allowance of spring pasture. The study was conducted from September to November of 2005. Twenty eight spring calving Friesian cows were assigned to four treatments: SP, grazing alone; PSC3, pasture plus $3 \mathrm{~kg} / \mathrm{d}$ of concentrate; PSC6, pasture plus $6 \mathrm{~kg} / \mathrm{d}$ and PSC9, pasture plus $9 \mathrm{~kg} / \mathrm{d}$ of concentrate. The cows were supplemented twice a day and managed under a strip grazing system. Weight and body condition were recorded individually each week. Milk production was recorded individually on a daily basis and composition was analyzed each week. Coccigeal blood samples from each cow were obtained each week after the morning milking and analyzed for $\beta \mathrm{OH}$-butyrate, NEFA, urea and albumin. Milk production for the SP, PSC2, PSC3 and PSC4 treatments were 28.1; 30.8; 31.2 and 28.8 1/day, respectively ( $P>0.05)$. The concentrations of protein or urea in milk were not affected by treatments. Grain supplementation decreased $(0.57 \%)$ milk fat concentrations $(\mathrm{P}<0.05)$, but the level of grain feeding had no effect among treatments $(\mathrm{P}>0.05)$. Plasma $\beta O H$-butyrate decreased $(\mathrm{P}<0.05)$ with concentrate supplementation; meanwhile plasma NEFA and urea were not affected by concentrate supplementation or by increasing the level of grain feeding, averaging $0.31 \mathrm{mmol} / 1$ and $32.0 \mathrm{~g} / 1$, respectively $(\mathrm{P}>0.05$ ). It can be concluded that under these experimental conditions increasing the level of concentrate supplementation did not modify milk production or composition, the live weight or the body condition on dairy cows on spring pasture receiving a high daily herbage allowance.

Palabras clave: vaca lechera, concentrado, pastoreo.

Key words: dairy cow, concentrate, pasture.

\section{INTRODUCCIÓN}

En el sur de Chile, la utilización directa de praderas permanentes es la principal fuente de alimento de las vacas destinadas a producción láctea (Balocchi y col 2002), sin embargo, la cantidad y la calidad de la pradera producida (elevado contenido de proteína cruda, un bajo porcentaje de materia seca y niveles medios de energía) no son constantes a través del año (FIA-UACH 1995, AFRC 1993). Además, las limitaciones que impone la pradera desde el punto de vista espacial y de sus características físicas (Hodgson 1990) impiden a los animales con medianos a altos niveles productivos alcanzar un elevado consumo de materia seca y que puedan satisfacer sus requerimientos nutricionales de energía a través del año (Delaby y col 2003). Es aceptado que la calidad nutricional de la pradera en primavera es la mejor en el año (FIA-UACH 1995) y que el uso de altas ofertas de pradera con el fin de maximizar el consumo voluntario a pastoreo conlleva a una baja eficiencia de utilización de ésta (McGilloway y

Aceptado: 06.05.2009.

\# Financiado por Proyecto FONDECYT 1030331.

* Casilla 567, Valdivia, Chile; rpulido@uach.cl
Mayne 1996). Sin embargo, este manejo de ningún modo permite que las vacas alcancen un consumo de alimento y de energía similar a lo logrado en vacas a pastoreo suplementadas con concentrado (Bargo y col 2003), por lo que es común el uso de alimentos suplementarios a fin de sobrellevar las limitaciones que presenta la pradera para una óptima productividad.

Es aceptado que diversos factores del animal, del alimento y del manejo afectan la respuesta productiva a la suplementación de vacas en pastoreo. Los factores dependientes del alimento base y del suplemento (disponibilidad y calidad) determinarán la magnitud en que la respuesta potencial podrá alcanzarse y la tasa de sustitución del forraje por alimento suplementario (Kellaway y Porta 1993). Entre los factores nombrados, la oferta de pradera por vaca por día y el nivel de concentrado tienen un gran efecto sobre el consumo total de alimento y sobre la respuesta en producción de leche (Peyraud y Delaby 2001), permitiendo que la energía adicional consumida y el mejoramiento del balance de energía (Coffey y col 2004) redujeran la movilización de tejido corporal. Sin embargo, la respuesta marginal en producción de leche tiende a disminuir cuando aumenta la oferta de pradera por vaca por día (Grainer y Mathews, 1989) y cuando aumenta el nivel 
de suplementación con concentrado (Robaina y col 1998). No obstante, existe limitada información sobre la cantidad adecuada de forraje que se debe ofrecer a las vacas lecheras posparto en primavera (Kennedy y col 2007).

La relación existente entre la respuesta de la producción de leche y las cantidades de concentrado es crucial para determinar el uso más efectivo de éste (Kellaway y Porta 1993). Los resultados experimentales muestran que las respuestas a la suplementación pueden ser lineales o curvas al aumentar el nivel de concentrados. En vacas lecheras de alta producción, la producción de leche a inicios de lactancia aumenta linealmente a medida que el consumo de MS de concentrado aumenta hasta $10 \mathrm{~kg} /$ día con una respuesta promedio de $1 \mathrm{~kg}$ leche $/ \mathrm{kg}$ de concentrado (Bargo y col 2002, Gibb y col 2002, Reis y Combs 2000). Sin embargo, en estas mismas vacas pero en lactancia tardía la producción de leche aumentaría a medida que la cantidad de concentrado aumenta, pero con una menor respuesta marginal por kilo de concentrado (Bargo y col 2003).

La principal fuente energética usada como suplemento en las explotaciones lecheras de la zona sur del país es el almidón de los granos de cereales, tales como el maíz, la avena y la cebada. Estos suplementos concentrados son caracterizados por modificar la proporción molar de los ácidos grasos volátiles en rumen, aumentando los precursores glucogénicos (principalmente propionato) y, por ende, influenciando el estatus del metabolismo energético de la vaca (Knowlton 1998).

Si bien la literatura es abundante en información del efecto del nivel de suplementación y la oferta de pradera en vacas a pastoreo sobre el consumo de alimento y la producción de leche (Bargo y col 2003, Peyraud y Delaby 2001), es necesario recabar más información respecto del efecto de niveles crecientes de concentrado en vacas en pastoreo con oferta generosa de pradera en primavera que potencien un consumo de alimento cercano al máximo sobre la respuesta productiva al comienzo de la lactancia (Delaby y col 2003, McEvoy y col 2008).

El objetivo de este trabajo fue evaluar el efecto de distintos niveles de suplementación con concentrado sobre el comportamiento productivo de vacas lecheras a pastoreo primaveral con alta oferta de pradera.

\section{MATERIAL Y MÉTODOS}

El ensayo se efectuó en el predio experimental "Vista Alegre", de propiedad de la Universidad Austral de Chile, ubicado 6 kilómetros al norte de la ciudad de Valdivia (39॰47'46"' y 39॰48'54' de Latitud Sur y 73²12'24' y 73 13 '13' de Longitud Oeste), desde septiembre hasta noviembre de 2005, por un período de 63 días. Se utilizaron 28 vacas Frisón Negro, que al inicio del estudio promediaban 56,4 \pm 4,7 días de lactancia, 4,1 $\pm 0,1$ partos, una producción láctea de 30,7 \pm 0,7 litros, una condición corporal de $2,3 \pm 0,1$ y un peso vivo de $538 \pm 13$ kilos (promedio $\pm \mathrm{DE}$ ). Las vacas fueron bloqueadas en cuatro grupos de siete vacas cada uno, de acuerdo a la producción de leche, días en leche y peso vivo. Los grupos se asignaron al azar a 4 tratamientos dietarios: SP, sólo pastoreo; PSC3, pastoreo más suplementación con 3 kg/día de concentrado; PSC6, pastoreo más suplementación con $6 \mathrm{~kg}$ /día de concentrado y PSC9, pastoreo más suplementación con $9 \mathrm{~kg} /$ día de concentrado, en un diseño continuo.

La pradera correspondió a una del tipo permanente mejorada, constituida principalmente por Lolium perenne $(70,4 \%)$, otras gramíneas $(14,4 \%)$, Trifolium repens $(4,6 \%)$, hoja ancha $(2,4 \%)$ y material muerto $(8,2 \%)$ (Bertín 2006), con uniformidad en edad y manejo de fertilización (fertilizada con $40 \mathrm{~kg} / \mathrm{ha}$ de nitrógeno previo al inicio del ensayo) y ubicada entre 300 y 600 metros de la sala de ordeña. Se utilizó un concentrado peletizado especialmente formulado para el ensayo, compuesto por un $94 \%$ de cebada, $4 \%$ de afrecho de raps y un $2 \%$ de melaza, siendo ofrecido en parcialidades iguales en cada ordeña según tratamiento. Sales minerales (Veterquímica S.A.) se ofrecieron junto al concentrado y también a libre disposición en saleros en el camino a la sala de ordeña, a razón de $250 \mathrm{~g} / \mathrm{vaca}$ al día. El agua se ofreció a libre disponibilidad, tanto en bebederos móviles en los potreros como en bebederos que se encontraban en la sala de ordeña. La ordeña se realizó dos veces al día, empezando a las 6:30 y 17:00 horas.

Cada una de las vacas fue individualizada por medio de collares numerados. La producción de leche se midió diaria e individualmente y se promedió cada semana para su análisis estadístico. Se tomaron muestras de leche consecutivamente en las ordeñas de la mañana y la tarde en un día de cada semana durante todo el ensayo. Semanalmente se registró el peso vivo de manera individual en la mañana después de cada ordeña, sin considerar destare. Finalmente, el cambio de peso vivo se calculó por regresión del peso vivo en el tiempo. Como elemento de medición se utilizó una balanza para $1.500 \mathrm{~kg}$, con sensibilidad de $0,1 \mathrm{~kg}$.

Las vacas se manejaron bajo un sistema de pastoreo rotativo en franjas, en donde cada tratamiento pastoreó en forma independiente una propia superficie de pastoreo (franja) y tuvo una rotación de potrero dos veces al día, inmediatamente posordeña. A cada vaca se otorgó una oferta de pradera de $36 \mathrm{~kg} /$ día de materia seca (MS), dividida en dos raciones (franjas) diarias, a fin de ofrecer una disponibilidad de pradera de al menos dos veces el consumo máximo esperado o ad libitum (Hodgson 1990). Se calculó superficie de pastoreo para cada tratamiento por el método de medición de la altura de la pradera prepastoreo, a través de una regresión lineal entre la altura de la pradera, obtenida cada día y para cada potrero, y la disponibilidad de la MS, ecuación previamente calculada y actualizada a lo largo del estudio, de acuerdo a lo señalado por Hodgson (1990). El mismo método se empleó para el pospastoreo, cuantificando así el residuo dejado por cada tratamiento. 
Para determinar las características degradativas de los alimentos se utilizaron tres vacas de raza Overo negro de dos años, fistuladas en el rumen con una cánula permanente de $11 \mathrm{~cm}$ de diámetro. Para la incubación se usaron bolsas de dacrón de $12 \mathrm{~cm}$ de largo por $5 \mathrm{~cm}$ de ancho para el concentrado y $22 \mathrm{~cm}$ de largo por $11 \mathrm{~cm}$ de ancho para la pradera, con tamaño de poros de $43 \mu \mathrm{m}$. En cada bolsa previa identificación se colocaron muestras con aproximadamente $5 \mathrm{~g}$ de MS.

\section{ANÁLISIS DE MUESTRAS}

Quincenalmente se tomó una muestra compuesta de las praderas pastoreadas por cada tratamiento y del concentrado utilizado, las que fueron analizadas en el Laboratorio de Nutrición Animal de la Universidad Austral de Chile. El contenido de MS se determinó mediante horno de ventilación a $60{ }^{\circ} \mathrm{C}$ por 48 horas, o estufa a $105^{\circ} \mathrm{C}$ por 12 horas (Bateman 1970); las cenizas totales (CT) mediante calcinación en mufla a $550-600{ }^{\circ} \mathrm{C}$ por 5 horas (Bateman 1970); la energía metabolizable (EM) a partir de regresión del valor "D” (Garrido y Mann 1981); el extracto etéreo (EE) mediante análisis proximal (Bateman 1970); la fibra detergente neutra (FDN) por digestión con detergente neutro (Van Soest y col 1991); la fibra detergente ácida (FDA) a partir de digestión con detergente ácido (AOAC 1996); y la proteína bruta (PC) mediante microkjeldhal (Bateman 1970). Las muestras de pradera se tomaron desde los $7 \mathrm{~cm}$ sobre el suelo, utilizando una tijera, para simular lo consumido por las vacas. La digestibilidad de los alimentos fue calculada a partir del valor "D" mediante el método de digestibilidad in vitro con licor ruminal de Tilley y Terry (1963) modificado por Goering y Van Soest (1972). La materia grasa, proteína y la urea de la leche se analizaron por espectrofotometría infrarroja (Foss 4300 Milko-scan; Foss Electric, Denmark), en el laboratorio de Cooprinsem, Osorno.

El proceso de degradación intrarruminal para los dos tipos de muestras tuvo los siguientes tiempos de incubación: 0, 2, 4, 8, 16, 24 y 48 horas, además incluyó un tiempo de incubación de 72 horas para la pradera. Luego de la incubación, las bolsas fueron retiradas del rumen y lavadas en lavadora con agua fría por cinco minutos sin centrifugar. Se hizo un mínimo de tres repeticiones por cada tiempo en cada vaca. Las bolsas de hora cero fueron sometidas al mismo procedimiento de lavado que los otros tiempos de incubación. Posterior al lavado, fueron secadas en un horno a $60{ }^{\circ} \mathrm{C}$ por 48 horas y pesadas, para obtener por diferencia la materia desaparecida. En los residuos de las bolsas se determinó la materia seca, la proteína cruda y la fibra detergente neutro, para calcular la tasa de desaparición de estos constituyentes nutricionales.

Para evaluar la dinámica de la degradación ruminal y calcular posteriormente la degradabilidad efectiva, los promedios de degradabilidad por cada tiempo se ajustaron a las ecuaciones propuestas por Orskov y McDonald (1979). Además se calculó la degradabilidad efectiva de acuerdo a la ecuación de McDonald (1981).
A partir del séptimo día del período experimental y posteriormente cada semana se obtuvieron muestras de sangre de todos los animales. Las muestras de $7 \mathrm{ml} \mathrm{de}$ sangre se obtuvieron mediante venopunción coccígea; $3 \mathrm{ml}$ fueron adicionados a un tubo con $\mathrm{NaF}$ para la determinación de la glucemia y $4 \mathrm{ml}$ fueron adicionados a un tubo con heparina sódica para la determinación de las concentraciones de $\beta \mathrm{OH}$-butirato, NEFA, urea y albúmina plasmática. Posteriormente, las muestras de sangre fueron centrifugadas por 10 minutos a $2.000 \mathrm{rpm}$ y el plasma fue alicuotado y congelado en microtubos de 1,5 $\mathrm{ml}$. Se determinaron las concentraciones plasmáticas de glucosa (GOD-PAP, Roche No 1448668), urea (GD UV cinético, Human, $\mathrm{N}^{\circ} 10521$ ) y albúmina (verde de bromo cresol, Human, $\mathrm{N}^{\mathrm{o}}$ 10560) en un autoanalizador Cobas

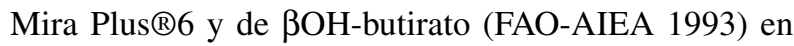
un espectrofotómetro Hitachi $4020^{\circledR}$.

\section{ANÁLISIS ESTADÍSTICO}

Los datos fueron sometidos a un análisis de varianza aleatorio continuo mediante la utilización de medidas repetidas, con un nivel de significancia del 5\%, empleando el programa MINITAB ${ }^{1}$. La comparación de las diferencias entre tratamientos se realizó mediante la prueba de Tukey. El modelo estadístico fue $y_{i j k l}=\mu+T_{\mathrm{i}}+C_{\mathrm{ij}}+$ $P_{\mathrm{k}}+T P_{\mathrm{ik}}+e_{j k l .}$, donde: $y_{i j k l}=$ variables dependientes; $\mu=$ intercepto general; $T_{\mathrm{i}}=$ el efecto fijo del tratamiento; $C_{\mathrm{ij}}=$ el efecto fijo de la vaca; $P_{\mathrm{k}}=$ el efecto fijo del período kth de observación; $T P_{\mathrm{ik}}=$ el efecto fijo de la intersección entre el tratamiento ith y el período kth de observación; $e_{j k l}=$ error residual.

\section{RESULTADOS}

\section{CARACTERÍSTICAS DE LOS ALIMENTOS Y MANEJO DE LA PRADERA}

El cuadro 1 muestra la composición química promedio de muestras quincenales de los alimentos utilizados en el estudio, observándose en el concentrado un alto nivel de digestibilidad y alta concentración de energía metabolizable. En la pradera destaca su alta concentración energética $(2,87$ $\mathrm{Mcal} / \mathrm{kg}$ ) y su concentración promedio de FDN (51\%). En el cuadro 2 se presentan las características de la cinética degradativa de los alimentos utilizados. Se observa que la degradabilidad de la materia seca y de la proteína cruda de la pradera mostró altos valores de la fracción soluble (a) y de la potencialmente degradable $(a+b)$.

\section{PRODUCCIÓN Y COMPOSICIÓN DE LA LECHE}

En el cuadro 4 se presenta la producción y la composición de la leche promedio según tratamiento. Se aprecia

MINITAB (2004) Release 14.12. Statistical Software. 
Cuadro 1. Composición nutricional del forraje y concentrado utilizados en el ensayo. Nutritional composition of the herbage and concentrates used in the trial.

\begin{tabular}{lcccc}
\hline & \multicolumn{2}{c}{ Concentrado $^{*}$} & \multicolumn{2}{c}{ Pradera } \\
\cline { 2 - 5 } & \multicolumn{2}{c}{$\mathrm{X} \pm \mathrm{DE} \pm \mathrm{DE}$} \\
\hline Materia seca (MS)\% & 87,3 & 0,96 & 15,9 & 3,02 \\
Cenizas totales (CT)\% & 3 & 0,55 & 7 & 2,46 \\
Proteína bruta (PC)\% & 13 & 0,98 & 22,4 & 4,67 \\
Extracto etéreo (EE)\% & 2,6 & 0,58 & 3,1 & 0,92 \\
Energía metabolizable (EM) Mcal/kg & 3,13 & 0,10 & 2,87 & 0,15 \\
Fibra detergente neutro (FDN)\% & 22,3 & 2,83 & 51 & 8,36 \\
Fibra detergente ácida (FDA)\% & 6,5 & 1,54 & 25,3 & 4,16 \\
IVDMD $^{1 \%}$ & 87,6 & 0,74 & 78 & 1,65 \\
\hline
\end{tabular}

${ }^{1}$ IVDMD $=$ Digestibilidad de la materia seca in vitro.

* = Concentrado formulado especialmente para este experimento por IANSAGRO S.A.

Cuadro 2. Constantes degradativas ruminales de la materia seca, proteína cruda y la fibra detergente neutro (FDN) para los alimentos utilizados en el ensayo.

Rumen degradation characteristics of dry matter, crude protein and neutral detergent fiber (NDF), from the food used in this trial.

\begin{tabular}{lccccc}
\hline \multicolumn{5}{c}{ Constantes de degradación ruminal $^{*}$} \\
\hline Alimento & $\mathrm{a}(\%)$ & $\mathrm{b}(\%)$ & $\mathrm{a}+\mathrm{b}(\%)$ & $\mathrm{c}$ & $\mathrm{DE}$ g/kg MS \\
\hline Concentrado & & Constantes degradativas de la materia seca & \\
Pradera consumida & 39,79 & 47,19 & 86,98 & 0,225 & 77,05 \\
\hline Concentrado & 52,26 & 42,71 & 94,97 & 0,072 & 75,58 \\
\hline Pradera consumida & \multicolumn{7}{c}{ Constantes degradativas de la proteína cruda } & 0,1029 & 78,90 \\
\hline Concentrado & 52,04 & 42,53 & 94,57 & 0,222 & 85,34 \\
Pradera consumida & 41,47 & 55,74 & 97,21 & & \\
\hline
\end{tabular}

* a = fracción soluble, a+b = degradación máxima potencial, c = tasa de degradación de la fracción b y DE = degradabilidad ruminal efectiva.

Cuadro 3. Manejo del pastoreo.

Grazing management.

\begin{tabular}{lcc}
\hline & Promedio & DE \\
\hline Carga animal (vacas/ha) & 3,08 & - \\
Oferta de pradera (kg MS/vaca/día) & 36,7 & 6,3 \\
Disponibilidad (kg MS/ha)* $^{*}$ & 2.893 & 417 \\
Residuo (kg MS/ha)* $^{*}$ & 1.726 & 239 \\
Utilización (\%) $^{*} 40,3$ & 3,8 \\
Altura promedio prepastoreo (plato) $^{1}$ & 24,5 & 4,7 \\
Altura promedio pospastoreo (plato) $^{1}$ & 17,1 & 2,1 \\
\hline
\end{tabular}

* = Ecuación de disponibilidad de pradera; $\mathrm{y}=737,5+88,1 \mathrm{x}\left(\mathrm{r}^{2}=0,85\right)$,

$\mathrm{y}=$ Fitomasa disponible; $\mathrm{x}=$ altura comprimida del plato.

${ }^{1}=$ Altura comprimida con plato medidor $(1 / 2 \mathrm{~cm})$. que no hubo diferencias significativas $(\mathrm{P}<0,05)$ en la producción de leche entre tratamientos y que ésta disminuyó significativamente menos en el grupo que recibió $3 \mathrm{~kg}$ de concentrado al día. No se observaron diferencias estadísticamente significativas en la concentración de la proteína y urea en leche. Sin embargo, sí se observó una disminución de materia grasa por efecto del nivel de suplementación $(\mathrm{P}<0,05)$.

\section{PESO VIVO Y CAMBIO DE PESO VIVO}

El peso vivo y la condición corporal de los animales según tratamiento se muestran en el cuadro 4 . No se observaron diferencias significativas en el peso vivo ni en la ganancia de peso vivo ni en el promedio de condición corporal de las vacas. 
Cuadro 4. Producción y composición de leche, peso vivo y condición corporal, y metabolitos sanguíneos y urinarios de las vacas durante el ensayo.

Production and composition of milk, body weight, body condition score, and blood and urinary metabolites of the cows during the trial.

\begin{tabular}{|c|c|c|c|c|c|}
\hline & \multicolumn{5}{|c|}{ Tratamientos } \\
\hline & SP & PSC3 & PSC6 & PSC9 & $\mathrm{P}$ \\
\hline \multicolumn{6}{|c|}{ Producción y composición de la leche } \\
\hline Leche (L/día) & $28,1^{\mathrm{a}}$ & $30,8^{\text {a }}$ & $31,2^{\mathrm{a}}$ & $29,1^{\text {a }}$ & 0,357 \\
\hline Proteína (\%) & $3,28^{\mathrm{a}}$ & $3,25^{\text {a }}$ & $3,22^{\text {a }}$ & $3,32^{\mathrm{a}}$ & 0,704 \\
\hline Grasa $(\%)$ & $3,86^{\mathrm{a}}$ & $3,32^{\mathrm{b}}$ & $3,36^{\mathrm{b}}$ & $3,19^{\mathrm{b}}$ & 0,008 \\
\hline Urea $(\mathrm{mg} / \mathrm{L})$ & $322^{a}$ & $322^{a}$ & $310^{\mathrm{a}}$ & $299^{\text {a }}$ & 0,303 \\
\hline \multicolumn{6}{|l|}{ Peso vivo y condición corporal } \\
\hline Peso vivo $(\mathrm{kg})$ & $535^{\mathrm{a}}$ & $564^{\text {a }}$ & $541^{\mathrm{a}}$ & $543^{\text {a }}$ & 0,773 \\
\hline Condición corporal (puntos) & $2,4^{\mathrm{a}}$ & $2,5^{\mathrm{a}}$ & $2,5^{\mathrm{a}}$ & $2,5^{\mathrm{a}}$ & 0,847 \\
\hline Cambio en peso vivo ( $\mathrm{kg} /$ día) & $0,46^{\mathrm{a}}$ & $0,61^{\mathrm{a}}$ & $0,77^{\mathrm{a}}$ & $0,85^{\text {a }}$ & 0,131 \\
\hline \multicolumn{6}{|l|}{ Metabolitos plasmáticos } \\
\hline ßOH-butirato (mmol/L) & $0,47^{\mathrm{a}}$ & $0,38^{\mathrm{b}}$ & $0,37^{\mathrm{b}}$ & $0,39^{\mathrm{b}}$ & 0,049 \\
\hline $\mathrm{NEFA}(\mathrm{mmol} / \mathrm{L})$ & $0,38^{\mathrm{a}}$ & $0,28^{\mathrm{a}}$ & $0,29^{\text {a }}$ & $0,30^{\mathrm{a}}$ & 0,200 \\
\hline Albúmina $(\mathrm{g} / \mathrm{L})$ & $33,4^{\mathrm{a}}$ & $32,1^{\mathrm{ab}}$ & $30,6^{\mathrm{b}}$ & $31,9^{\mathrm{b}}$ & 0,047 \\
\hline Urea $(\mathrm{mmol} / \mathrm{L})$ & $5,61^{\mathrm{a}}$ & $5,27^{\mathrm{a}}$ & $5,52^{\mathrm{a}}$ & $5,35^{\mathrm{a}}$ & 0,453 \\
\hline
\end{tabular}

Letras distintas en una fila señalan diferencias significativas $(\mathrm{P}<0,05)$.

\section{METABOLITOS PLASMÁTICOS}

Como se indica en el cuadro 4, las concentraciones plasmáticas de $\beta \mathrm{OH}$-butirato y de albúmina fueron menores en el grupo sólo a pastoreo con respecto a los grupos suplementados $(\mathrm{P}<0,05)$, pero no diferentes entre tratamientos suplementados $(\mathrm{P}>0,05)$. La concentración de NEFA no mostró diferencias ni por efecto de la suplementación ni entre grupos suplementados.

\section{DISCUSIÓN}

\section{CARACTERÍSTICAS DE LOS ALIMENTOS Y MANEJO DE LA PRADERA}

Las praderas permanentes del sur de Chile durante primavera poseen en promedio un $15,2 \%$ de MS; $11,5 \%$ de CT; $17,8 \%$ de PC; 3,2\% de EE; $27,9 \%$ de FDA y 2,55 $\mathrm{Mcal} / \mathrm{kg}$ (FIA-UACH 1995). Al comparar los valores referenciales con los del cuadro 1 se encuentran valores similares para la MS, EE y FDA y distintos para CT, PC y EM. Por lo tanto, la pradera presentó un alto valor nutritivo, especialmente para la concentración de EM y PC. La concentración de FDN para una pradera de buena calidad, según Kolver y Müller (1998), es aquella que fluctúa entre un 40 y $50 \%$, alcanzando para este ensayo a un $51 \%$, concordando con lo reportado anteriormente y con ensayos realizados en similares condiciones por Pulido y col (2007).

Las características de la cinética degradativa se muestran en el cuadro 2. Se observa que la degradabilidad de la MS de la pradera mostró altos valores de fracción soluble a tiempo cero y que produjo una degradabilidad potencial máxima de un 94,9\%. Al respecto, Van Vuuren y col (1991) informaron que las gramíneas perennes (Lolium perenne) a comienzos de primavera presentan altos valores de la fracción soluble, los que se encuentran asociados con bajos tenores de FDN y altas proporciones de hoja/tallo. La dinámica degradativa del FDN para la fracción potencialmente degradable arrojó valores de 67,3\%, indicando que el FDN de esta pradera presenta una gran disposición para ser degradado, lo que podría generar una entrega de energía para los microorganismos ruminales.

Con respecto a la PC, se encontró una alta fracción soluble y degradabilidad efectiva $(41,5 \%$ y $97,2 \%$, respectivamente), evidenciando un contenido proteico de alta degradabilidad, característico de una pradera con una gran proporción de hojas en relación a tallos (Pulido y Leaver 2000). Lo anterior es coincidente con lo establecido por Hodgson (1990), en el sentido de que las vacas seleccionan material verde en preferencia a tallos o material muerto.

La degradabilidad efectiva de la materia seca y la proteína cruda del concentrado amiláceo $(39,8 \%$ y 50,0\%, respectivamente) se encuentran de acuerdo con lo descrito para un suplemento con materias primas similares (Sayers y col 2003).

Los parámetros degradativos de las muestras de forraje son concordantes con los estimados para forrajes templados en el Reino Unido (Agricultural and Food Research Council 1993) y de Australia (Wales y col 1999). Finalmente, se debe tener presente que dichos parámetros son de difícil comparación entre ensayos, en virtud de las 
metodologías aplicadas en la preparación de los alimentos, animales utilizados, condiciones ruminales, tipo de lavado, entre otras.

En el cuadro 3 se observa que la carga animal fue de 3,08 , valor usualmente encontrado en este tipo de ensayos (Bargo y col 2003, Pulido y col 2007), debido a una alta disponibilidad de forraje primaveral y al uso de un elevado número de vacas en una superficie relativamente pequeña. La utilización fue de 40,3\% y la oferta de pradera promedió los $36,7 \mathrm{~kg} / \mathrm{MS} / \mathrm{vaca} /$ día, valor que estuvo dentro del rango objetivo de este ensayo (generosa oferta de pradera). Por su parte, la altura promedio de la pradera fue mantenida sobre los $11 \mathrm{~cm}$, lo que asociado a la baja presión de pastoreo podría indicar, según Hodgson (1990), que el consumo de pradera estaría en los valores máximos para vacas lecheras a pastoreo, lo que se lograría sólo en cortos períodos durante la primavera, cuando la oferta y la calidad de la pradera son muy buenas.

\section{PRODUCCIÓN Y COMPOSICIÓN DE LA LECHE}

Es aceptado que la respuesta a la suplementación en este tipo de ensayos (de corto plazo) depende de la tasa de sustitución, de la calidad del suplemento en relación a la calidad de la pradera, y de la partición de la energía adicional consumida entre producción de leche y ganancia de peso corporal (Bargo y col 2003, Kellaway y Porta 1993). En el cuadro 4 se aprecia que los valores de producción de leche, proteína láctea y urea en leche no presentaron diferencias significativas entre tratamientos $(\mathrm{P}>0,05)$; sin embargo, la materia grasa de la leche disminuyó por efecto del nivel de suplementación $(\mathrm{P}<0,05)$, concordante con lo señalado por Bargo y col (2002). Es sabido que la respuesta en producción de leche por kilo de concentrado disminuye a causa de aumentar la disponibilidad de pradera (Grainer y Mathews 1989) y por efecto de aumentar el nivel de suplementación con concentrado (Robaina y col 1998). En este ensayo, las condiciones generosas de oferta de pradera en promedio de $36 \mathrm{~kg} / \mathrm{MS} / \mathrm{vaca} /$ día y alta calidad de ésta permitieron que el consumo de pradera promedio en las vacas sin suplementación alcanzara a los 17,6 kg de materia seca y generara una alta tasa de sustitución en la medida que se aumentara el nivel de suplementación, lo que explicaría el hecho de que el consumo total de MS fuera similar entre tratamientos, a excepción por PSC3, que logró el mayor consumo comparado con PSC9 (Riquelme y Pulido 2008), y no se encontraran diferencias en producción con los grupos suplementados.

Por lo tanto, los resultados de este ensayo serían coincidentes con lo señalado por Peyraud y Delaby (2001) en el sentido de que la respuesta marginal tiende a disminuir arriba de 3 a $4 \mathrm{~kg}$ /día de concentrado, cuando las vacas lecheras son de moderado mérito genético, se encuentran a mediados de lactancia o la cantidad y calidad de la pradera no es limitante.

\section{PESO VIVO Y CAMBIO DE PESO VIVO}

El peso vivo y el cambio de peso vivo de los animales, según tratamiento, se muestra en el cuadro 4. No se observaron diferencias significativas en el peso vivo, el cambio de peso vivo ni en la condición corporal de las vacas. Estos resultados son coincidentes con lo presentado por Bargo y col (2002), Pulido y Leaver (2001), los que tampoco obtuvieron diferencias de peso vivo ni condición corporal al suplementar vacas en pastoreo con niveles de suplementación dentro del rango de lo utilizado en este ensayo. Baker y Leaver (1986) señalan que la ausencia de efecto en el peso vivo promedio por tratamiento podría ser explicado por el corto período de duración del ensayo o por los efectos confundidos del llenado intestinal al momento del pesaje, los cuales afectarían de menor manera al calcular el cambio de peso vivo. Por lo tanto, sería esperable que el cambio de peso vivo y la condición corporal mejoraran en los grupos con mayor nivel de suplementación con concentrado.

\section{METABOLITOS PLASMÁTICOS}

Las concentraciones plasmáticas de $\beta \mathrm{OH}$-butirato fueron mayores en el grupo sólo a pastoreo con respecto a los grupos suplementados $(\mathrm{P}<0,05)$, pero no diferentes entre tratamientos suplementados (cuadro 4). Similares resultados obtuvieron Noro y col (2006) en vacas en pastoreo con condiciones similares de calidad y disponibilidad de pradera primaveral y suplementadas con $6 \mathrm{~kg} / \mathrm{vaca} /$ día de un concentrado similar al utilizado en este ensayo. Por lo tanto, estos resultados indicarían que en las vacas suplementadas con concentrado amiláceo, sin importar el nivel de suplementación, la fuente energética de rápida degradación ruminal favorecería el balance energético del animal, al mejorar la eficiencia de utilización del nitrógeno de la pradera por parte de los microorganismos ruminales (Bargo y col 2002, Coffey y col 2004, Sinclair y col 2000), aumentar la glucemia y disminuir la concentración de urea en sangre y en consecuencia ajusta el metabolismo intermediario energético y proteico (Sinclair y col 2000). La concentración de NEFA no mostró diferencias ni por efecto de la suplementación ni entre grupos suplementados.

Las concentraciones plasmáticas de albúmina fueron similares entre los grupos sólo a pastoreo y los grupos PSC3 y PSC6 $(\mathrm{P}<0,05)$, no diferentes entre tratamientos suplementados y mayor en SP comparado con PSC9 $(\mathrm{P}<0,05)$. Al igual que el trabajo de Noro y col (2006), todos los tratamientos mantuvieron las concentraciones de albúmina dentro de los rangos de referencia para la especie (29 a $41 \mathrm{~g} / \mathrm{L}$ ), lo que indicaría que los animales se encontraban en condiciones adecuadas en cuanto al metabolismo de proteínas. 


\section{RESUMEN}

Se realizó un experimento con el objeto de evaluar el efecto de distintos niveles de suplementación con concentrado, sobre el comportamiento productivo de vacas lecheras a pastoreo primaveral, recibiendo una generosa oferta de pradera. Se usaron 28 vacas Frisón Negro, las que fueron asignadas a los siguientes tratamientos: SP, sólo pastoreo; PSC3, pastoreo más $3 \mathrm{~kg}$ de concentrado; PSC6, pastoreo más $6 \mathrm{~kg}$ de concentrado y PSC9, pastoreo más $9 \mathrm{~kg}$ de concentrado. Las vacas fueron suplementadas dos veces al día y manejadas en pastoreo rotativo. En cada vaca el peso vivo y la condición corporal fueron registrados semanalmente y la producción láctea diariamente. La composición de la leche se analizó semanalmente. Semanalmente a cada animal se tomaron muestras de sangre para determinar las concentraciones plasmáticas de $\beta O H$-butirato, NEFA, urea y albúminas. La producción láctea fue de 28,1; 30,8; 31,2 y 28,8 L/día, para los tratamientos SP, PSC2, PSC3 y PSC4 respectivamente $(\mathrm{P}>0,05)$. Las concentraciones lácteas de proteína o urea no fueron afectadas por los tratamientos. La suplementación disminuyó en un $0,57 \%$ las concentraciones de grasa en leche $(\mathrm{P}<0,05)$, pero no tuvo efecto el aumento del nivel de suplementación. Las concentraciones plasmáticas de $\beta \mathrm{OH}$-butirato disminuyeron $(\mathrm{P}<0,05)$ en los tratamientos suplementados. Sin embargo, las concentraciones plasmáticas de NEFA y de urea $(0,31 \mathrm{mmol} / \mathrm{L}$ y $32,0 \mathrm{~g} / \mathrm{L}$, respectivamente) no fueron modificadas ni por la suplementación ni por el aumento de la suplementación con concentrado. Se puede concluir bajo las condiciones de este ensayo que la suplementación con distintos niveles de concentrado no afectó la producción ni la composición de leche, el peso vivo ni la condición corporal en vacas lecheras a pastoreo.

\section{REFERENCIAS}

Agricultural and Food Research Council. 1993. Energy and protein requirements of ruminants. An advisory manual prepared by the AFRC Technical Committee on Responses to Nutrients. CAB International, Wallingford, UK.

Association of official analytical chemists. 1996. Official methods of analysis. $16^{\text {th }}$ ed. AOAC, Arlington, VA, USA.

Baker AM, JD Leaver. 1986. Effect of stocking rate in early season on dairy cow performance and sward characteristics. Grass Forage Sci 41, 333-340.

Balocchi O, R Pulido, J Fernández. 2002. Comportamiento de vacas lecheras en pastoreo con y sin suplementación de concentrado. Agri Téc 62, 87-98.

Bargo F, LD Muller, JE Delahoy, TW Cassidy. 2002. Milk response to concentrate supplementation of high producing dairy cows grazing at two pasture allowances. J Dairy Sci 85, 1777-1792.

Bargo F, LD Muller, ES Kolver, JE Delahoy. 2003. Invited review: Production and digestion of supplemented dairy cows on pasture. J Dairy Sci 86, 1-42.

Bateman J. 1970. Nutrición animal. Manual de métodos analíticos. Centro Regional de Ayuda Técnica, México.

Bertin R. 2006. Efecto de dos niveles de fitomasa de pre y pospastoreo sobre características productivas de una pradera permanente en primavera. Memoria de Título, Facultad de Ciencias Agrarias, Universidad Austral de Chile, Valdivia, Chile.

Coffey MP, G Simm, JD Oldham, WG Hill, S Brotherstone. 2004. Genotype and diet effects on energy balance in the first three lactations of dairy cows. J Dairy Sci 87, 4318-4326.

Delaby L, JL Peyraud, N Foucher, M Guillaume. 2003. The effect of two contrasting grazing managements and level of concentrate supplementation on the performance of grazing dairy cows. Anim Res 52, 437-460.

FAO- IAEA, Food and Agriculture Organization - International Atomic Energy Association. 1993. Nutritional Metabolite Kit Protocols. Ed. Joint FAO/IAEA Division, Animal Production and Health Section.
FIA-UACH. 1995. Composición de alimentos para el ganado en la zona sur. Universidad Austral de Chile. Fundación Fondo de Investigación Agropecuaria (eds). Ministerio de Agricultura, Editorial Universitaria, Valdivia, Chile.

Garrido O, E Mann. 1981. Composición química, digestibilidad y valor energético de una pradera permanente de pastoreo a través del año. Memoria de Título, Facultad de Ciencias Agrarias, Universidad Austral de Chile, Valdivia, Chile.

Gibb MJ, CA Huckle, R Nuthall. 2002. Effects of level of concentrate supplementation on grazing behaviour and performance by lactating dairy cows grazing continuously stocked grass swards. Anim Sci 74, 319-335.

Goering H, P Van Soest. 1972. Análisis de fibra de forraje. Universidad Agraria La Molina, Lima, Perú.

Grainer C, GL Mathews. 1989. Positive relation between substitution rate and pasture allowance for cows receiving concentrates. Aust $J$ Exp Agric 29, 355-360.

Hodgson J. 1990. Grazing Management. Science into Practice. Longman Scientific and Technical. Essex, England, Pp 203.

Kellaway R, S Porta. 1993. Feeding concentrates: Supplements for dairy cows. Dairy Research and Development Corporation, Glen Iris, Victoria, Australia.

Kennedy E,M O’Donovan, FP O'Mara, JP Murphy, L Delaby. 2007. The Effect of Early-Lactation Feeding Strategy on the Lactation Performance of Spring-Calving Dairy Cows. J Dairy Sci 90, 3060-3070.

Knowlton KF, TE Dawson, BP Glenn, GB Huntington, RA Erdman. 1998. Glucose metabolism and milk yield of cows infused abomasally or ruminally with starch. J Dairy Sci 81, 3248-3258.

Kolver ES, LD Muller. 1998. Performance and nutrient intake of high producing Holstein cows consuming pasture or a total mixed ration. J Dairy Sci 81, 1403-1411.

McDonald IM. 1981. A revised model for the estimation of protein degradability in rumen. J Agric Sci Camb 96, 251-252.

McEvoy M, E Kennedy, JP Murphy, TM Boland, L Delaby, M O'Donovan. 2008. The Effect of Herbage Allowance and Concentrate Supplementation on Milk Production Performance and Dry Matter Intake of Spring-Calving Dairy Cows in Early Lactation. J Dairy Sci 91, 1259-1269.

McGilloway DA, CS Mayne. 1996. The importance of grass availability for the high genetic merit dairy cow. In: Garnsworthy PC, Wiseman J, Haresign W (eds). Recent Advances in Animal Nutrition 1996. Nottingham University Press, Nottingham, UK, Pp 135-169.

Noro M, V Vargas, RG Pulido, F Wittwer. 2006. Efecto del tipo de concentrado sobre indicadores sanguíneos del metabolismo de energía y de proteínas en vacas lecheras en pastoreo primaveral. Arch Med Vet 38, 227-232.

Orskov ER, I McDonald. 1979. The estimation of protein degradability in the rumen from incubation measurements weighted according to rate of passage. J Agric Sci Camb 92, 499-503.

Peyraud JL, L Delaby. 2001. Ideal concentrate feeds for grazing dairy cows responses to supplementation in interaction with grazing management and grass quality. In: Garnsworthy PC, Wiseman J (eds). Recent Advances in Animal Nutrition. Nottingham University Press, Nottingham, UK, Pp 203

Pulido R, JD Leaver. 2000. Degradabilidad ruminal del forraje disponible en las praderas y el aparentemente consumido por vacas lecheras. Pesq Agropec Bras 35, 1003-1009.

Pulido RG, JD Leaver. 2001. Quantifying the influence of sward height, concentrate level, and initial milk yield on the milk production and grazing behaviour of continuously stocked dairy cows. Grass Forage Sci 56, 57-67.

Pulido RG, S Berndt, P Orellana, F Wittwer. 2007. Effect of the source of carbohydrate in concentrate on the performance of high producing dairy cows during spring grazing. Arch Med Vet 39, 19-26. 
Reis RB, DK Combs. 2000. Effects of increasing levels of grain supplementation on rumen environment and lactation performance of dairy cows grazing grass-legume pasture. J Dairy Sci 83, 28882898.

Riquelme C, RG Pulido. 2008. Efecto del nivel de suplementación con concentrado sobre el consumo voluntario y comportamiento ingestivo en vacas lecheras a pastoreo primaveral. Arch Med Vet 40, 243-249.

Robaina AC, C Grainger, P Moate, J Taylor, J Stewart. 1998. Responses to grain feeding by grazing dairy cows. Aust J Exp Agric 38, 541549.

Sayers HJ, CS Mayne, CG Bartram. 2003. The effect of level and type of supplement offered to grazing dairy cows on herbage intake, animal performance and rumen fermentation characteristics. Anim Sci 76, 439-454.
Sinclair KD, LA Sinclair, JJ Robinson. 2000. Nitrogen metabolism and fertility in cattle: I. Adaptative changes in intake and metabolism to diets differing in their rate of energy and nitrogen release in the rumen. J Anim Sci 78, 2659-2669.

Tilley JMA, RA Terry. 1963. A two stage technique for in vitro digestion of forage crops. J Br Grassl Soc 18, 104-111.

Van Soest PJ, J Robertson, B Lewis. 1991. Methods for dietary fiber, neutral detergent fiber, and nonstarch polysaccharides in relation to animal nutrition. J Dairy Sci 74, 3583-3595.

Van Vuuren AM, S Tamminga, RS Ketelaar. 1991. In sacco degradation of organic matter and crude protein of fresh grass (Lolium perenne) in the rumen of grazing dairy cows. J Agric Sci Camb 116, 429-436.

Wales WJ, DW Dellow, PT Doyle. 1999. Degradabilities of dry matter and crude protein from perennial herbage and supplements used in dairy production systems in Victoria. Aust J Exp Agric 39, 645-656. 